\title{
Gap Junctions between Striatal Fast-Spiking Interneurons Regulate Spiking Activity and Synchronization as a Function of Cortical Activity
}

\author{
Johannes Hjorth, ${ }^{1,3}$ Kim T. Blackwell, ${ }^{4}$ and Jeanette Hellgren Kotaleski ${ }^{1,2,3}$ \\ ${ }^{1}$ Computational Biology, School of Computer Science and Communication, Royal Institute of Technology, Albanova University Centre, 10691 Stockholm, \\ Sweden, ${ }^{2}$ Nobel Institute for Neurophysiology, Department of Neuroscience, and ${ }^{3}$ Stockholm Brain Institute, Karolinska Institutet, 17177 Stockholm, \\ Sweden, and ${ }^{4}$ Molecular Neuroscience Department, Krasnow Institute for Advanced Study, George Mason University, Fairfax, Virginia 22030
}

Striatal fast-spiking (FS) interneurons are interconnected by gap junctions into sparsely connected networks. As demonstrated for cortical FS interneurons, these gap junctions in the striatum may cause synchronized spiking, which would increase the influence that FS neurons have on spiking by the striatal medium spiny (MS) neurons. Dysfunction of the basal ganglia is characterized by changes in synchrony or periodicity, thus gap junctions between FS interneurons may modulate synchrony and thereby influence behavior such as reward learning and motor control. To explore the roles of gap junctions on activity and spike synchronization in a striatal FS population, we built a network model of FS interneurons. Each FS connects to 30-40\% of its neighbors, as found experimentally, and each FS interneuron in the network is activated by simulated corticostriatal synaptic inputs. Our simulations show that the proportion of synchronous spikes in FS networks with gap junctions increases with increased conductance of the electrical synapse; however, the synchronization effects are moderate for experimentally estimated conductances. Instead, the main tendency is that the presence of gap junctions reduces the total number of spikes generated in response to synaptic inputs in the network. The reduction in spike firing is due to shunting through the gap junctions; which is minimized or absent when the neurons receive coincident inputs. Together these findings suggest that a population of electrically coupled FS interneurons may function collectively as input detectors that are especially sensitive to synchronized synaptic inputs received from the cortex.

\section{Introduction}

The striatum, the input stage of the basal ganglia, is populated by several types of neurons (Kawaguchi et al., 1995). Striatal fastspiking interneurons (FS) strongly inhibit the numerous medium spiny projection neurons (MS) that are the output neurons of the striatum (Bennett and Bolam, 1994; Koós and Tepper, 1999; Bolam et al., 2000; Tepper et al., 2004). Both neuron types receive glutamatergic input from cortex and thalamus, as well as dopaminergic input from substantia nigra compacta (Bolam et al., 2000; Bar-Gad et al., 2003; Graybiel, 2005; Ding et al., 2008). The FS interneurons are interconnected through gap junctions (electrical synapses) into sparsely connected networks (Koós and Tepper, 1999; Galarreta and Hestrin, 2001b) that provide inhibition to MS neurons. Experiments show that FS activity may directly modulate MS spike timing in vitro (Koós and Tepper, 1999).

\footnotetext{
Received Dec. 18, 2008; revised March 24, 2009; accepted March 26, 2009.

J.H. and J.H.K. acknowledge support from the Swedish Research Council and the Parkinson's Foundation. K.T.B. was supported by National Institutes of Health (R01AA16022) and the Human Frontiers Science Program. We thank Sten Grillner, Gilad Silberberg, and Anders Lansner for useful discussions, and also John Wolf for providing the original MS model code.

Correspondence should be addressed to Johannes Hjorth, Computational Biology, School of Computer Science and Communication, Royal Institute of Technology, Albanova University Centre, 10691 Stockholm, Sweden. E-mail: hjorth@kth.se.

D01:10.1523/JNEUROSCI.6031-08.2009

Copyright $\odot 2009$ Society for Neuroscience $\quad 0270-6474 / 09 / 295276-11 \$ 15.00 / 0$
}

The timing of MS neuron spikes is important because they influence plasticity in the presence of dopamine (Schultz, 1998; Reynolds and Wickens, 2002). For example, stimulation of the cortex induces spike-timing-dependent plasticity (STDP) at corticostriatal synapses (Fino et al., 2005, 2008; Pawlak and Kerr, 2008; Shen et al., 2008). Also, the calcium increase due to an action potential is larger for shorter delays between the up-state onset and first MS spike (Kerr and Plenz, 2004). Thus, synchronization of FS interneurons by gap junctions may increase FSto-MS inhibition and more effectively delay spikes or control spike timing in MS neurons, thereby modulating MS neuron plasticity.

Previous theoretical and modeling studies have shown that gap junctions can affect synchronization (Chow and Kopell, 2000; Traub et al., 2001; Amitai et al., 2002; Nomura et al., 2003; Pfeuty et al., 2003; Gibson et al., 2005). Experimental studies in both neocortical and hippocampal networks have shown that gap junctions increase synchronicity of firing. Gap junctions can also play a role in maintaining spike synchronization in a heterogeneous population of neurons (Traub et al., 2001). Despite this convincing role of gap junctions in synchronization, neither model nor experimental studies have demonstrated an increase in synchrony in striatal FS interneurons when driven by synaptic inputs. Instead recent in vivo experiments show uncoordinated changes in firing rate of striatal FS interneurons in behaving animals (Berke, 2008). 
In this paper we use computational modeling to study the effect of gap junctions between striatal FS interneurons. The FS interneurons are activated with either synaptic input or somatic current injections. Simulation experiments suggest that, while the gap junctions synchronize the neurons weakly, their dominating effect is overall activity reduction, leading to a reduction in firing rate in the whole network. Nonetheless, when the network is activated by correlated synaptic input, the reduction in firing frequency becomes lower or disappears. This could allow the FS network to act as a coincidence detector, able to distinguish between correlated and uncorrelated inputs.

\section{Materials and Methods}

FS network. Striatal fast-spiking (FS) interneurons were modeled using a previously published model (Kotaleski et al., 2006) implemented in GENESIS (Bower and Beeman, 1998). This 127-compartment model has a soma connected to three primary dendritic branches, which divide into secondary and tertiary branches. Fast sodium channels and three potassium channels, $\mathrm{Kv}_{3.1 / 3.2}, \mathrm{Kv}_{1.3}$, and $\mathrm{K}_{\mathrm{A}}$, reproduce action potential shape and firing patterns measured experimentally (Blackwell et al., 2003). To avoid artifacts due to a network of homogeneous neurons, the compartment length and potassium channel $\mathrm{K}_{\mathrm{A}}$ conductance were varied by $\pm 50 \%$ using a uniform distribution for each compartment. This leads to a heterogeneous network of cells, with variations in the current-frequency $(I-F)$ curves, shown in Figure $1 A$.

The FS model was extended by the addition of gap junctions, modeled as resistive elements, between the neurons, as illustrated in Figure $1 B$. Experimentally measured cortical gap junction conductance (Galarreta and Hestrin, 2002) and striatally measured FS interneuron coupling coefficient (Galarreta and Hestrin, 2001b; Tepper et al., 2004) were used to tune FS interneuron gap junction conductance. The coupling coefficient is defined as the fraction of a voltage deflection that reaches the coupled neuron as measured in the soma (Galarreta and Hestrin, 2001b). Figure $1 C$ shows the coupling coefficient for long current pulses (steady state) and spikes (transient) as a function of proximal gap junction conductance. Gap junctions in conjunction with the capacitive and resistive properties of the membrane act as low-pass filters (Galarreta and Hestrin, 1999, 2001b; Connors and Long, 2004), preferentially transmitting slow signals, such as longer current injections, better than action potentials. Even at high conductances the spike is considerably filtered when transmitted across the gap junction. A conductance of $0.5 \mathrm{nS}$ is within the higher experimental range of measured gap junction conductances (Galarreta and Hestrin, 2002) and is used in the model unless otherwise stated. The coupling coefficient increases slightly with depolarization and is maximal at around $-55 \mathrm{mV}$, after which it decreases. This is because the neuron's input resistance varies with voltage. Placing the gap junctions on the primary dendrites results in a coupling coefficient (as measured at resting potential) within the upper range measured in the striatum [Koós and Tepper (1999): 3-20\%] and is used in the simulations below.

The number of gap junctions and size of the local FS network were determined by experimental observations. Each MS neuron receives input from $55 \%$ of the nearby striatal FS interneurons (Tecuapetla et al., 2007), and at most 4-27 converging on the same MS neuron (Koós and Tepper, 1999). The probability of gap junctions between pairs of nearby FS neurons has been estimated at 30-40\% [Koós and Tepper (1999): 2 of 6 pairs; Tepper et al. (2004): extended to 3 of 7 pairs]. Based on these estimates a 10 FS neuron network, used for the initial study, corresponds to the FS network seen by a postsynaptic MS. Randomly connecting the neurons with one gap junction on each of three primary dendrites gives each FS interneuron three gap junctions (Fig. 1D), and gives a connection probability of $33 \%$, consistent with experiments. The FS interneurons were not interconnected additionally by GABAergic synapses; though striatal and cortical FS interneurons share their developmental origin, the striatal FS neurons appear to be lacking GABAergic connections between them (Koós and Tepper, 1999). Likewise, there appear to be fewer gap junctions between FS interneurons in striatum than in cortex (Galarreta and Hestrin, 2001b).
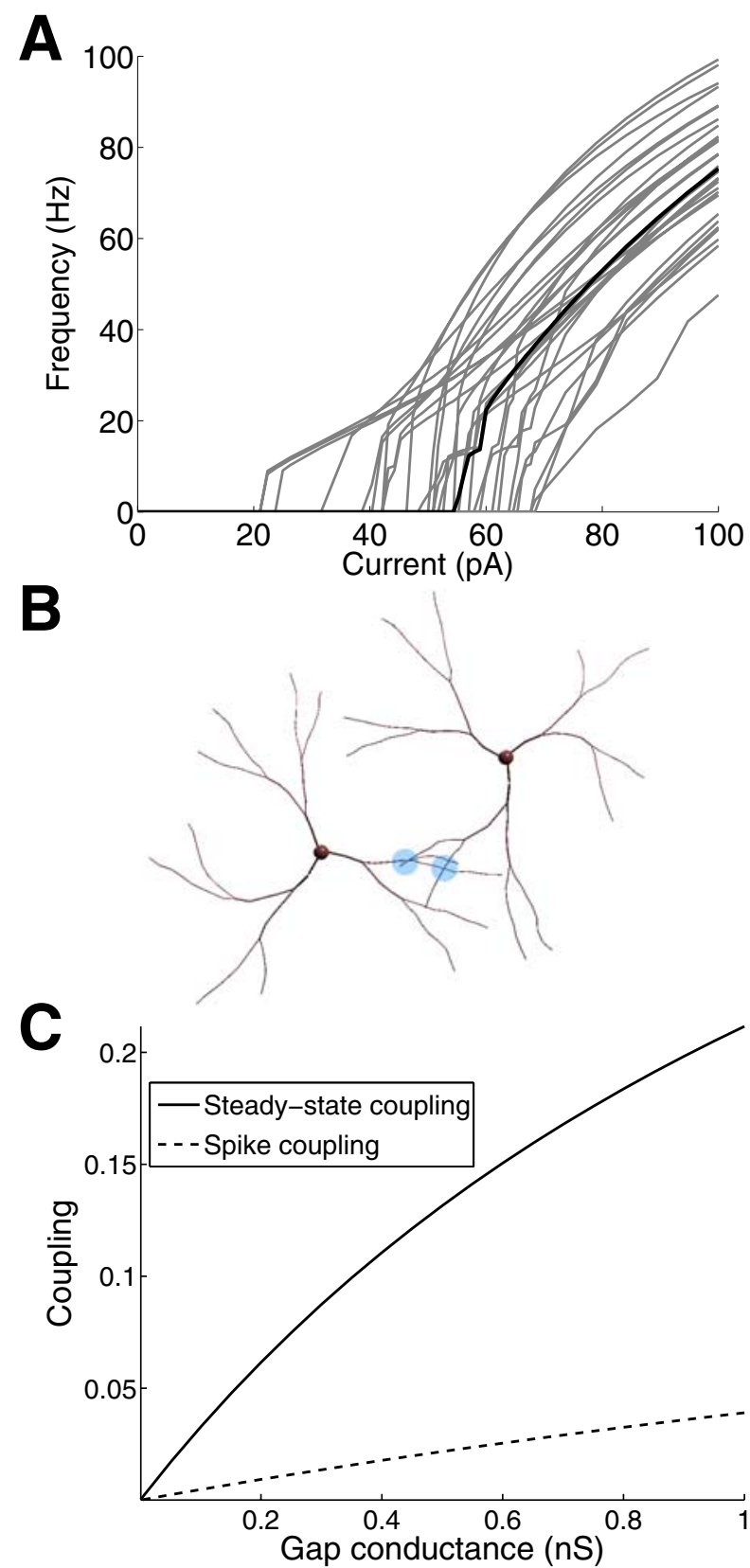

D
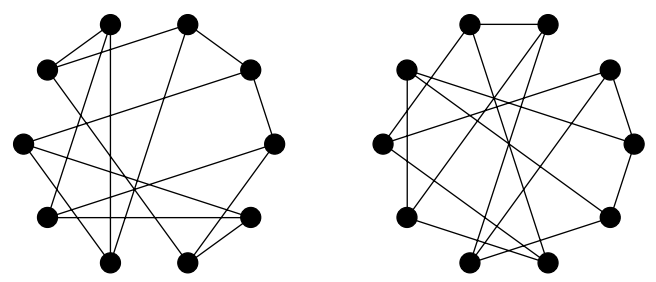

Figure 1. Model of striatal FS interneuron network with 10 neurons. $A, I-F$ curves for the FS interneurons in the network. To avoid homogeneous populations, compartment length and $K_{A}$ conductance have been varied. The I-F curve of the original model (Kotaleski et al., 2006) is marked in black. $\boldsymbol{B}$, Illustration of gap junction connections between two detailed FS neurons (not to scale). $\boldsymbol{C}$, Coupling coefficient as a function of the gap junction conductance for steady state current injection versus spikes. The spike is triggered by a very brief current pulse. Gap junctions behave as a low pass filter and thus the coupling coefficient is lower for spikes. In both cases, resting potential was $-63 \mathrm{mV}$ for both neurons, and single cell input resistance was $477 \mathrm{M} \Omega$. Both current injection and recording was somatic; spikes were generated by $1 \mathrm{~ms}, 0.2 \mathrm{nA}$ injection. $\boldsymbol{D}$, Example of networks of randomly electrically coupled FS interneurons. Only the soma of each neuron is shown, but the electrical connections are on primary dendrites. Each neuron has gap junctions to three neighbors, which gives a coupling probability of one-third between nearby neurons. 
Verification using a larger FS network. The results from the 10 neuron network were verified in a larger $125 \mathrm{FS}$ network, which corresponds to a cube of striatal tissue with $0.5 \mathrm{~mm}$ sides. In the simulations the FS neurons were placed on a grid with an average distance of 135-150 $\mu \mathrm{m}$ between them. This is based on the estimated density of 300-400 FS/ $\mathrm{mm}^{3}$ in human caudate and putamen (Kalanithi et al., 2005). The number of gap junctions per FS neuron was constrained by the connection probability of one-third (Koós and Tepper, 1999). For proximal gap junctions there are at least 10 neighboring FS within dendritic connection range (i.e., neurons up to $200 \mu \mathrm{m}$ apart) and for distal gap junctions there are at most 45 (i.e., $300 \mu \mathrm{m}$ apart). This corresponds to a lower limit of 3 and an upper limit of 15 gap junctions per FS neuron. The average number of gap junctions in the simulations was varied between 0 (no gap junctions) and 16. This is in accordance with recent estimates of gap junction densities (Fukuda, 2009) which give 1-24 gap junctions per FS neuron, with a modal value of 5-7. Each gap junction was placed independently and the probability that any given neighbor received a connection was proportional to the overlapping volume of the dendritic tree with the parent neuron, as inspired by Wickens et al. (2007).

Simulated synaptic input. The synaptic input in the FS model is similar to what is measured experimentally in organotypic cocultures (Blackwell et al., 2003), because of the availability of quantitative data on input frequency and amplitude. The model has 127 AMPA synapses, evenly distributed throughout the cell, and 93 GABA synapses, distributed on soma, and primary and secondary dendrites. Each synapse has an input frequency of $\sim 2 \mathrm{~Hz}$ during up-state-like periods of activity, which results in a total AMPA input of $282 \mathrm{~Hz}$ and GABA input of $207 \mathrm{~Hz}$ (Kotaleski et al., 2006). In the model when down states are simulated, each synapse has an input frequency of $1 / 20$ of the up-state input. In sleeping or anesthetized animals and in cocultures, the input alternates between up and down states (Wilson and Kawaguchi, 1996; Plenz and Kitai, 1998; Stern et al., 1998; Kasanetz et al., 2006); however, in awake animals up-state-like activity dominates (Kasanetz et al., 2002; Mahon et al., 2006). Thus, for the study of synchronization and shunting in FS networks, only up-state input was used unless otherwise stated. In other cases where alternating up and down states were used, the results reported are similar for continuous up states, because the FS neuron has such short time constants that there is very little memory of previous activation. The AMPA and GABA inputs were assumed to be independent from one another in the absence of experimental data. If this assumption is lifted, and correlations are introduced between AMPA and GABA, qualitative findings are the same, but there is a moderate decrease in baseline firing frequency of the neurons for a given synaptic activation frequency. No attempt was made to segregate the corticostriatal and thalamostriatal pathways, neither temporally nor spatially. Similarly the GABAergic inputs from globus pallidus and intrastriatal sources were grouped together.

Corticostriatal axons have been found to make up to six synapses on a single FS interneuron (Ramanathan et al., 2002), implying that several synapses can be activated in a correlated manner. In the earlier model (Kotaleski et al., 2006) the correlation measure was generated in the same way as in Rudolph and Destexhe (2001). In the present model, MATLAB (version r2007b, MathWorks) was used to pregenerate a complete set of input spikes for a single FS interneuron. One Poisson distributed spike train was generated for the AMPA synapses and another for the GABA synapses. To introduce correlations in the FS input, the corresponding synapses randomly drew spikes (with replacement) from the shared pool of spikes with probability $P=1 / n$, where $n=N-\sqrt{c}(N-1), N=$ number of synapses, and $c=0.5$. Inputs to each FS interneuron in the network were independent unless explicitly stated; thus, the correlation, $c$, within a neuron was 0.5 (see supplemental Fig. S2, available at www. jneurosci.org as supplemental material), but the correlation between neurons was zero. The rationale is that nearby MS neurons in vivo have correlated up states, but uncorrelated fluctuations due to inputs within the up state (Kincaid et al., 1998; Stern et al., 1998; Kitano et al., 2001). To investigate the effect of between-neuron input correlation, an additional, shared set of input spikes was created. The population's input correlation was then varied by changing the fraction of the neuron's input taken from the shared input set. The scripts necessary to run both the $10 \mathrm{FS}$ and
125 FS networks are available for download at ModelDB (http://senselab.med.yale.edu/ModelDB/).

Analysis of spike synchronization. To investigate synchronization, joint peristimulus time histograms (JPSTHs) (Palm et al., 1988; Aertsen et al., 1989) were constructed for FS interneuron pairs receiving alternating up-state/down-state input. The periods were considered to be triggered by the start of the up states. The JPSTH reveals changes in the synchronization during the duration of an up state, with points on the diagonal corresponding to synchronous spikes for the neuron pair. To study quantitatively the effect of gap junctions on the synchronization, crosscorrelograms were generated for each directly coupled neuron pair in the 10 -cell network, and then averaged over the network. The same simulations were rerun without gap junctions as a reference.

The effect of gap junctions on the spike pattern was classified into three cases; removal of spikes due to shunting, spikes triggered by neighboring spikes and spikes appearing spontaneously. The comparison is between an FS population with and without gap junctions. For each spike in the reference network devoid of gap junctions, if no spike is within $\Delta t$ in the trace from the network with gap junctions, the spike is considered removed. Conversely, for each spike in the network with gap junctions, if there is no spike within $\Delta t$ in the reference network, the spike is considered added. To further evaluate the added spike, the traces from neurons connected with gap junctions were analyzed. If there were no spikes in those coupled neurons when they were uncoupled (i.e., in the reference network) at least $\Delta t$ before the spike, then the spike was considered to be spontaneous, otherwise it was spike triggered. This analysis accounts for all spike changes; summing them together resulted in the measured reduction in total number of spikes. Analyses were repeated for $\Delta t$ between 5 and $20 \mathrm{~ms}$.

\section{Results}

Gap junctions affect synchrony and reduce firing frequency

To illustrate the effect of gap junctions on spiking activity and synchrony, a pair of FS interneurons was investigated first. Each of the multicompartmental FS interneurons received independent synaptic input (Kotaleski et al., 2006), resulting in spiking activity as during up-state or awake periods. Spiking patterns in the two reference neurons were compared with simulations in which the two FS interneurons were coupled with a gap junction.

Figure $2 \mathrm{~A}$ shows an example voltage trace for both the reference (top) and gap junction coupled (bottom) case, when the two FS interneurons receive up-state or awake-state input. The addition of a gap junction changes the spike timing, but also changes the number of spikes. In some cases a new spike is triggered in the neighboring neuron (Fig. $2 \mathrm{~B}$, left); in other cases the presence of a gap junction causes the removal of an FS spike (Fig. $2 B$, right).

The proportion of synchronous spikes increases with gap junction conductances as illustrated in Figure $2 C$ for several time windows, from 5 to $20 \mathrm{~ms}$. Synchrony within these time windows would allow for inhibitory FS inputs to interact or sum in a postsynaptic MS neuron, since their IPSPs then overlap [Koos et al. (2004), their Fig. 2]. Though spike synchrony is observed in the pair of FS interneurons, the effect is quite weak at physiological gap junction conductances [mainly $<0.5 \mathrm{nS}$ (Galarreta and Hestrin, 2002)].

In Figure $2 D$ the relative increase in spike synchronization is illustrated further using an JPSTH during $250 \mathrm{~ms}$ up-state periods. Figure $2 D 1$ shows that spikes do not synchronize in the reference FS pair devoid of gap junctions, whereas Figure 2D2 shows an increase in spikes occurring within $5 \mathrm{~ms}$ of each other when gap junctions are simulated. This is represented by the increase in density of points on the diagonal.

Though the effect of gap junctions is easy to analyze and understand in a two neuron network, a larger network allows for coupling probabilities and interactions more similar to in vivo. Therefore, simulations were performed by providing indepen- 

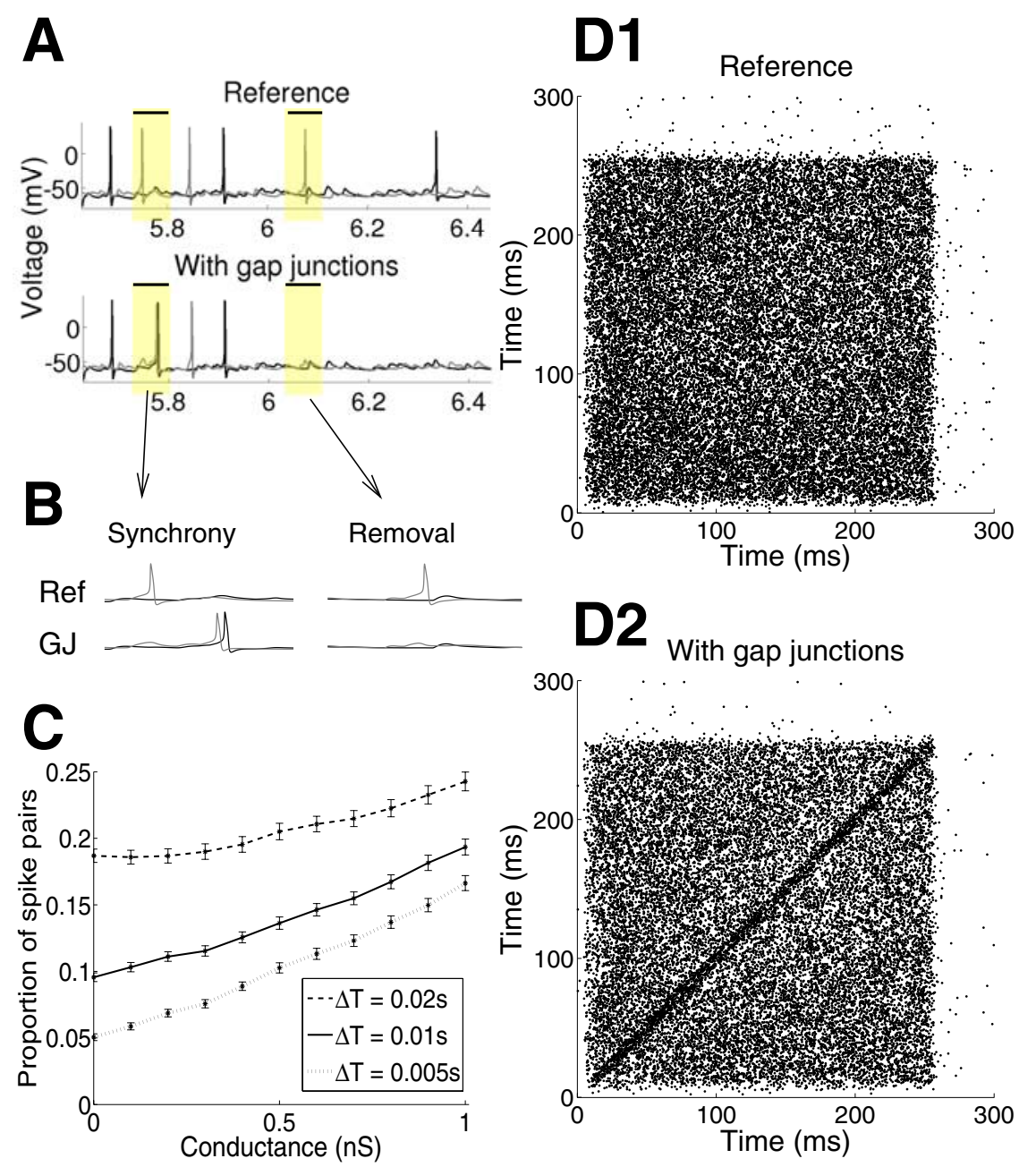

Figure 2. Role of gap junctions for synchronization in networks of two FS interneurons. $\boldsymbol{A}$, Example voltage traces for two neurons without (Reference) or with proximal gap junctions (conductance $0.5 \mathrm{nS}$ ). The same synaptic input is repeated in both simulations. Note the change in spike number and spike timing. $\boldsymbol{B}$, Zoomed in version of the traces in $\boldsymbol{A}$. In the left subgraph a spike appears later in the trace with gap junctions and in addition triggers a spike in the neighboring cell. The right subgraph shows the disappearance of a spike instead. C, Proportion of synchronized spikes as a function of the conductance of the gap junctions. A spike is considered synchronous with another spike in an electrically coupled neuron if it appears within time $\Delta t$. D, JPSTH for two reference FS interneurons (D1) and for the FS interneurons coupled with a gap junction conductance $0.5 \mathrm{nS}$ (D2). The diagonal represents synchronized spiking.

dent synaptic input to each of the 10 FS interneurons in the network. Cross correlograms were constructed by averaging the cross-correlations calculated between each pair of neurons in the network. For reference, simulations were repeated and the crosscorrelation was calculated for a network devoid of gap junctions but receiving identical input as before.

Figure $3 A$ shows that gap junctions produce some synchronization in the network (visible as a peak at $0 \mathrm{~ms}$ with width approximately $\pm 5 \mathrm{~ms}$ ), but the dominant feature is a reduction in overall firing frequency. All interspike intervals, binned at $1 \mathrm{~ms}$, show a decrease. The synchronized peak of the black (with gap junctions) cross-correlogram also lies below the base line of the cross-correlogram for the reference network (gray) devoid of gap junctions. As the gap junction strength is increased, two effects are observed: the proportion of synchronous spikes increases (as seen in Fig. 2C) and the overall activity in the FS network is reduced (Fig. $3 B$ ). As demonstrated below, this spike reduction is seen when the network is activated by synaptic inputs, and is not necessarily seen in FS populations activated by current injections (further explained below).
The moderate spike synchronization appearing in the network is not widespread; instead only directly coupled FS interneurons synchronize. Spike pairs between directly coupled FS interneurons appear more frequently than those between neurons only indirectly coupled (Fig. 3C). The latter appears only at levels comparable to what would be expected by chance, as illustrated by shuffling the traces of the directly coupled neurons and recalculating the spike pairs. This indicates that there is no global synchronization due to gap junctions in a small network of $10 \mathrm{FS}$ interneurons, receiving simulated synaptic awake-state input, and connected with $30-40 \%$ probability with a gap junction to each of the other cells in the network.

Both the shunting behavior and the minimal spike synchronization were verified in a larger 125 FS network (see supplemental Fig. S1, available at www. jneurosci.org as supplemental material). Simulations were performed with uncorrelated input in a large network either with or without gap junctions. A crosscorrelogram generated from all pairs of neurons revealed no synchronization, independent of the presence or absence of gap junctions, but firing frequency was reduced in the connected case. If only the interactions between directly coupled neighbors are measured, or a subnetwork is characterized, a small synchronization peak is apparent, as observed in the $10 \mathrm{FS}$ network (results not shown). Nonetheless, there is no global synchronization in the larger network. Thus, the results using a 10 FS neuron network are not due to network size.

\section{Mechanisms underlying the changes in spiking patterns}

Depending on the synaptic input, the addition of gap junctions can remove, delay, or even add spikes (Fig. $2 A$ ). Despite the fact that the current always flows away from the more depolarized neuron, a range of different spike changes can occur as illustrated in Figure 4. The left column shows a reference FS interneuron pair that is devoid of gap junctions, and the right column displays the same neuron pair with gap junctions on proximal dendrites $(0.5 \mathrm{nS})$. If an FS interneuron that originally spiked as a result of synaptic inputs is electrically coupled to a neuron that does not receive any input, the charge lost to the neighbor can bring the first neuron below threshold and thus prevent spiking (Fig. 4A). The picture changes if the simulations are repeated, but this time the neighbor also receives some subthreshold depolarizing synaptic input. The addition of a gap junction now leads to the transfer of some charge as before from the first to the second neuron, but slightly less than before since the second neuron is also depolarized. Thus the first neuron may still spike, but since the neighboring neuron also received synaptic input, the additional current through the gap junction brings the second neuron above threshold (Fig. 4B). Thus, depending on the degree of activation of nearby neurons, the addition of gap 

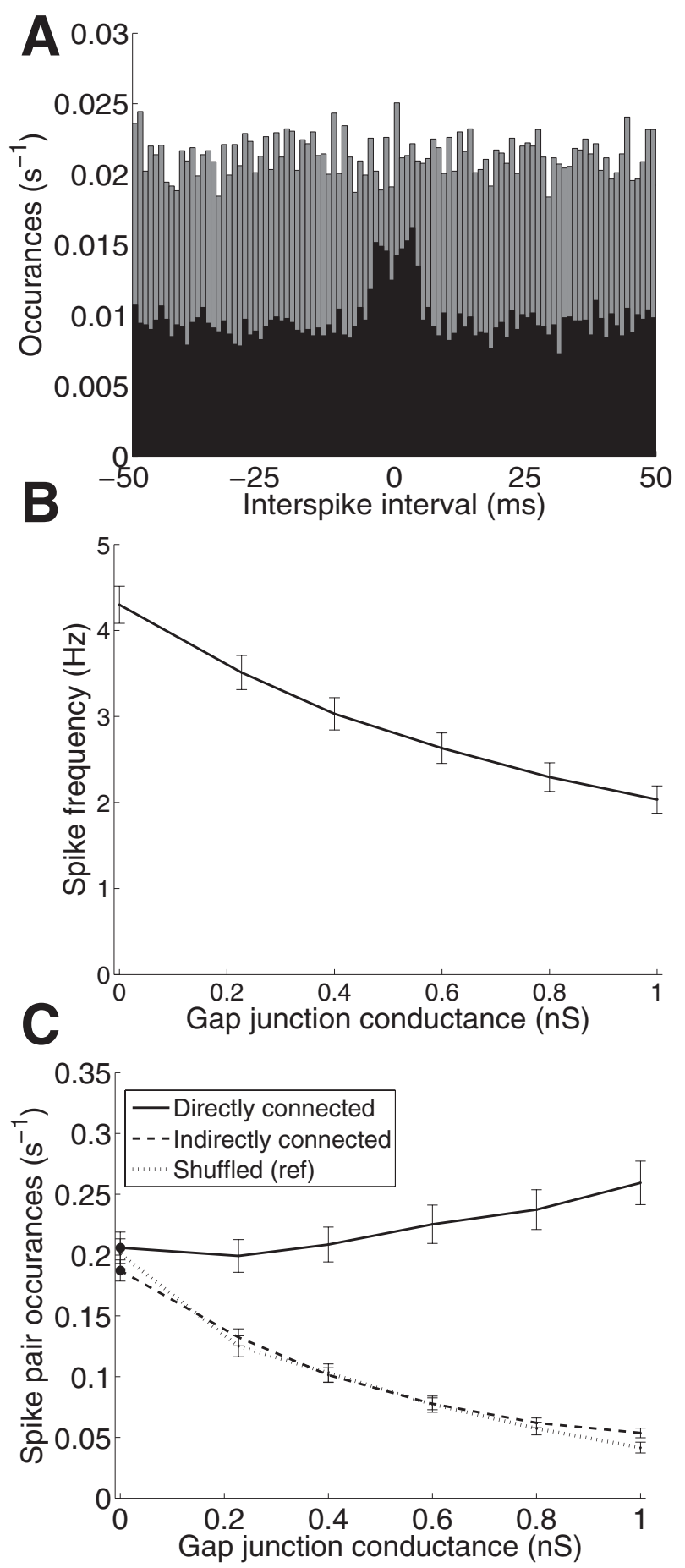

Figure 3. Influence of gap junctions on network spiking activity in a $10 \mathrm{FS}$ network. A, Cross correlogram shows increase in synchrony due to gap junctions for the FS network. The black bars show results for the electrically coupled network (proximal gap junctions, $0.5 \mathrm{nS}$ ), with a relatively higher rate of synchronous spikes at $\pm 5 \mathrm{~ms}$; the gray bars show the uncoupled reference case, which has a higher rate of spike pairs at all interspike time intervals (bin size $1 \mathrm{~ms}$ ) (compare with supplemental Fig. S1D, available at www.jneurosci.org as supplemental material). $\boldsymbol{B}$, Spike frequency as a function of gap junction conductance, showing the decrease in firing as the conductance increases for neurons receiving synaptic input. The reduction is due to loss of charge (shunting) through the gap junctions. C, The occurrences of spike pairs in directly coupled (solid line) or indirectly coupled (dashed line) neurons spiking within $\Delta t$ equals $5 \mathrm{~ms}$ of each other. Increased absolute numbers of synchronized spikes are limited to the directly electrically coupled neurons. The curve for the nondirectly coupled neurons follows what would be expected by chance, as illustrated by the close resemblance with the shuffled version of the coupled neuron case (where direct interactions mediated by gap junctions have been destroyed, but changes in correlation due to a change in firing frequency remain). junctions can both add or remove spikes. Figure $4 C$ shows that there does not have to be a spike in either neuron for gap junctions to add a new spike. Here the two neurons are activated one after the other, neither reaching threshold. The addition of gap junctions to this scenario transfers charge from the first neuron activated to the second neuron, which is then able to spike. In rare cases this spike may in turn evoke a spike in the first neuron also.

A quantification using a similar classification as illustrated in Figure $4 A-C$ can be applied to the modeled 10 FS network when activated by up-state synaptic inputs. Figure $4 D$ shows that overall the reduction in spikes (solid line; case A) is larger than the addition of spikes, and spikes appearing spontaneously (dashed line; case C) due to shifting of charge between electrically connected nonspiking neurons are more common than those triggered by spikes in neighboring neurons (dash-dotted line; case B). As gap junction conductance is increased, more spikes are shunted away than added, resulting in a successively larger net reduction of activity (dotted line).

\section{Highly correlated input maintains spiking activity}

The reduction in spike frequency is a novel finding and has not been reported in cortical FS interneuron networks. To investigate the source of this difference, simulations are performed using current injection instead of synaptic activation. Since the current shunted through the gap junctions is dependent on the voltage difference between the electrically coupled neurons, shunting should be minimal if neighboring neurons receive identical input. Conversely, shunting should be evident if neurons receive different amounts of current injection.

Figure $5 A$ shows how the $I-F$ curve, produced using somatic current injections, for one FS model neuron depends on the activation level in a neighboring identical neuron when they are connected with a gap junction. If both neurons are injected with the same current, there is no change in firing frequency due to the presence of the gap junctions, because with identical cells and identical inputs there is no shunting in either direction due to symmetry. However if the neighboring neuron receives less current (50\%, dashed line; no current, gray line; or hyperpolarizing currents, gray dashed line), then the first neuron will experience a reduction in firing. As the driving current of the first neuron increases, the dependence on the neighboring neuron's degree of activation decreases. This is due to the shape of the $I-F$ curve for the single FS interneuron: as the current increment needed to increase the firing frequency (e.g., $1 \mathrm{~Hz}$ ) increases higher up on the curve, shunting has a diminished effect on reducing firing frequency. Sodium inactivation and activation of Kv3.1/3.2 affect the shape of the $I-F$ curve in the model. When the sodium and potassium have smaller (faster) time constants, the spike initiation and repolarization are faster; thus, the slope of the $I-F$ curve is steeper and shunting decreases less with higher current injection compared with the control case.

To test the prediction that nonsynchronous synaptic input leads to spike reduction in the FS network while synchronous (or more coincident) synaptic input does not, a 10 FS interneuron network was simulated with various correlations (for definition of correlation, see Materials and Methods) among the synaptic inputs to different neurons. Figure $5 B$ shows that the reduction in firing frequency is higher when the input has low or no correlation between the electrically coupled FS interneurons. For a successive increase in the coincidence of activated synapses between the neighboring cells in the network, the shunting decreases and finally disappears. Consistent with these results, Figure $5 C$ illustrates that the outward current through the gap junction just 

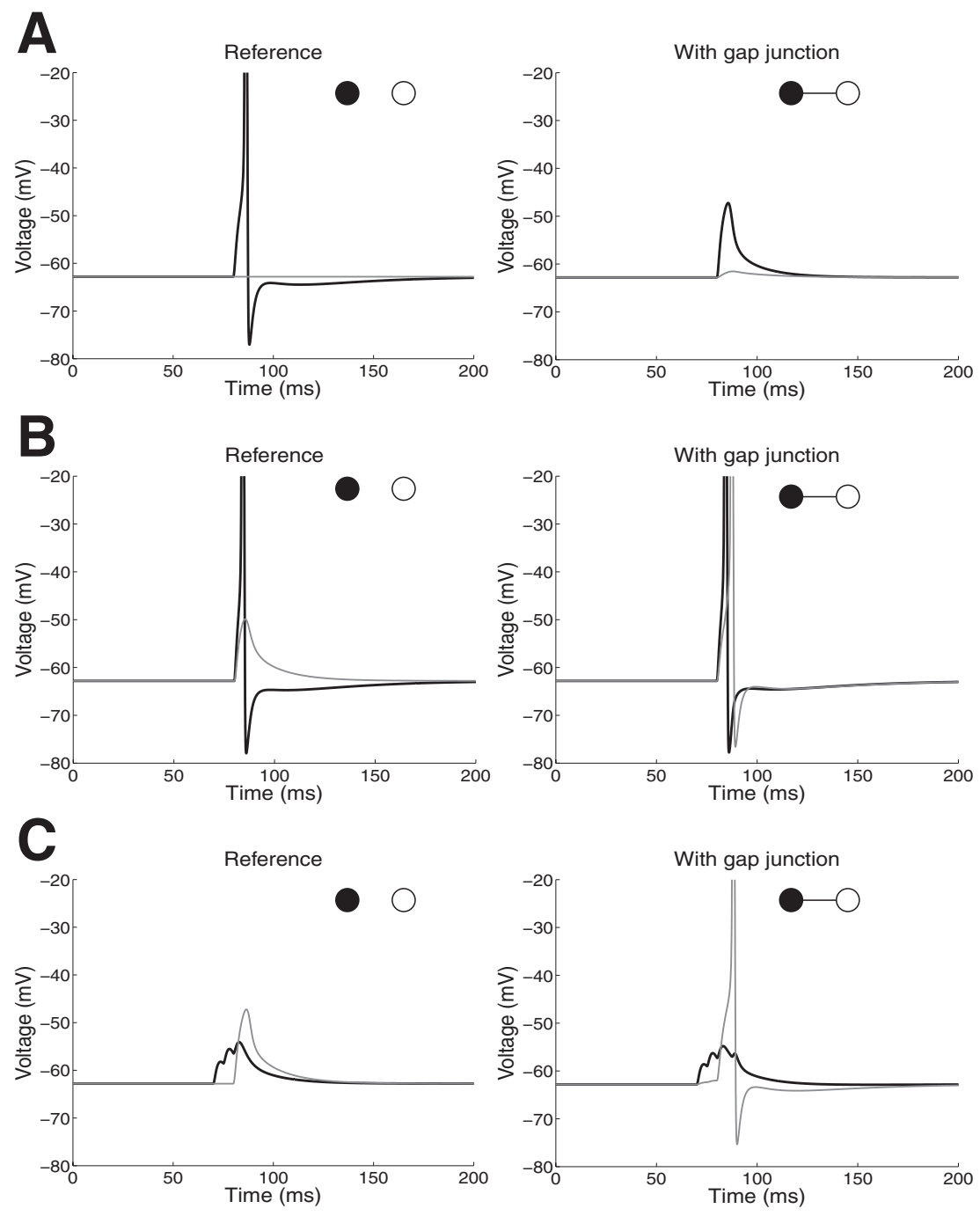

D

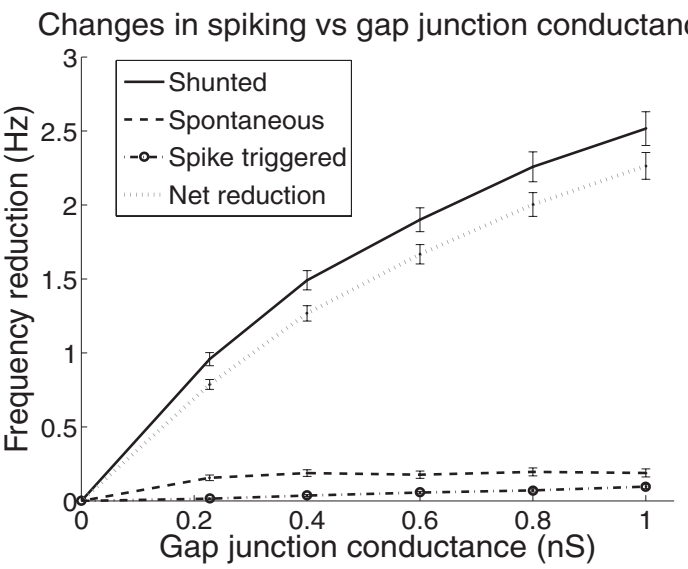

Figure 4. Mechanisms of spike appearance and disappearance in the electrically coupled networks. $\boldsymbol{A}$, Removal of a spike. In the electrically coupled case the neuron receiving synaptic input loses charge to its nondepolarized neighbor, resulting in a subthreshold response in both neurons. $\boldsymbol{B}$, Spike-induced triggering of a synchronized spike. The addition of gap junctions can sometimes lead to a sharing of the depolarization with the neighboring neuron, converting its subthreshold depolarization into a spike. C, Addition of a spike due to preceding depolarization of coupled neighboring neurons. First one neuron is depolarized by synaptic input, but not enough to cause a spike. In the presence of gap junctions some of this depolarization is transferred to the neighboring neuron, which brings it closer to threshold, sometimes allowing it to spike when it simultaneously receives additional synaptic input. D, Change in spikes caused by the addition of gap junctions. Solid trace shows the spikes shunted away (case A), dashed line those appearing spontaneously (case C), dash-dotted lines the spikes that are triggered by a spike in a neighboring neuron (case B), and gray dotted trace shows the net reduction in spikes. Note that the addition of spikes (cases B and C) is less common than the removal of spikes, producing a reduction in overall spiking activity with gap junctions. before a spike is increased for lower input correlations, making it harder for the FS interneurons to spike. This result does not depend on the method used to change input correlation. Similar qualitative and quantitative effects are seen if all cells in the network are activated at the same times and at the same locations in the dendritic tree. Nonsynchronous inputs are then produced by jittering the activation times at each synapse (see supplemental Fig. S3, available at www.jneurosci.org as supplemental material). Thus, the novel finding of a decrease in spike frequency due to gap junctions is due to lack of correlation among synaptic inputs compared with typical current injection protocols.

To further demonstrate the role of input correlation, and to document that the difference between our results and prior results is due to the use of uncorrelated synaptic activation, simulations of spike synchronization are repeated in response to current injection. For constant current injections above threshold the FS neurons spike regularly and the neighbors are also continuously depolarized, which means that the current losses through gap junctions are minimal, as in Figure $5 \mathrm{~A}$ (uppermost curve). This suggests that the synchronizing effect of gap junctions would dominate during current injections. Simulations show that, similar to results in Figure $2 D$ for the two neuron network, gap junctions synchronize networks of FS neurons driven by current injection (Fig. $5 D$ ). In networks activated with current injections spike frequency can even increase with an increased gap junction conductance (supplemental Fig. S4A, available at www.jneurosci.org as supplemental material), opposite to that seen with synaptic activation. Therefore, the observation that gap junctions reduce spike frequency more so than enhancing synchrony is due to the uncorrelated nature of synaptic input, not due to unusual properties of striatal FS interneurons.

\section{Spike frequency reduction due to gap junctions depends on synaptic input intensity} Just as spike frequency reduction depends on current injection amplitude, it also depends on synaptic input frequency. As a neuron approaches spike threshold the voltage difference across the gap junction increases, leading to an increased outward current through the gap junction. Similarly, increased synaptic input frequency implies more membrane potential fluctuations, producing a larger average voltage difference across the gap junction (Fig. 
$6 B)$ and thus more shunting on average. Thus, an increase in synaptic inputs is predicted to increase spike frequency reduction by these mechanisms. However, this increase in voltage across the gap junction is sublinear (Fig. $6 \mathrm{~B}$, inset), because when inputs arrive more frequently the degree of simultaneous depolarization also increases, reducing the shunting. To evaluate these competing mechanism, simulations were performed to see whether spike frequency reduction decreased with an increase in input frequency, similar to current injection, or increased due to larger voltage differences.

Figure $6 A$ shows that, similar to the result with current injection, the fraction of spikes removed by the presence of gap junctions decreases with increased synaptic input frequency. Before a spike, the neuron is close to threshold and the connected neighbor is also more depolarized due to the increased synaptic input frequency. This results instead in a smaller voltage difference across the gap junctions just before the action potential and thus a decreased outward current for higher input frequencies, as shown in the spike centered plot in Figure 6C. Consequently, the increase in removed spikes is slower than the increase in total spike frequency. In addition, at higher input frequencies a spike is triggered more easily in a neighboring neuron (Fig. 6D, dash-dotted line). The number of added spikes increases proportionally more than the number of spikes removed; thus the net reduction in firing frequency levels out for high synaptic input frequencies. A constant spike frequency reduction translates into a decrease in the proportion of removed spikes because total spike frequency increases with synaptic input frequency.

The relative decrease in spike reduction with increased input synaptic activity may be accompanied by an increase in synchrony in networks with higher synaptic input. Figure $6 E$ shows that the increase in number of synchronized spike pairs (within 5 $\mathrm{ms}$ ) per neuron pair in the 10 neuron network with gap junctions is greater (solid line) for higher input frequencies, than what is predicted by chance for the same spike frequencies (see shuffled traces, dashed line). If the analysis is reduced to only the directly coupled neighbors, spike synchronization even overcomes the shunting and the average number of spike pairs is higher (dotted line) than that in the network devoid of gap junctions (dashdotted line).

\section{Correlation detection}

What is the possible function for a population of electrically coupled FS interneurons, given that gap junctions reduce firing frequency with uncorrelated inputs, and synchronize firing in response to correlated inputs? The final set of simulations evaluates the response of an FS network to a transient increase in correlation, as may occur during a behavioral task. Figure $7 A$ shows that a striatal FS network of 10 neurons with gap junctions is able to detect rapid changes in correlations between the synaptic inputs

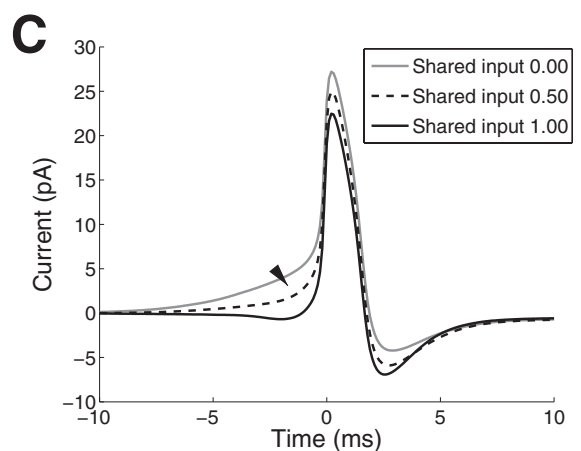

D

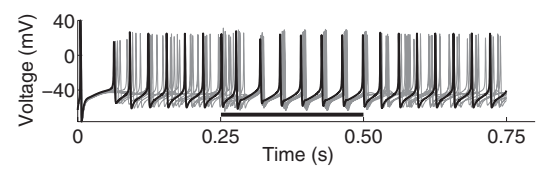

Figure 5. Spike frequency reduction depends on input coherence between connected neurons. $A, I-F$ curve for one FS interneuron connected to an identical neighboring FS interneuron with a gap junction. The neighboring neuron receives identical rrent injection (solid line), half amplitude (dashed line), no current (gray), or hyperpolarizing current (gray dashed). The firing presence of gap junctions, and desynchronizes when they are removed. Note that the same result is achieved with varied FS excitability and identical current injections to all neurons (data not shown).

to the different neurons. The FS network nearly doubled its firing during a short period $(20 \mathrm{~ms})$ of correlated synaptic inputs to neighboring neurons. Note that an FS network devoid of gap junctions would have the same spike frequency throughout because synaptic input frequency is not altered. This indicates that gap junctions allow the FS network to detect correlated activation patterns across the population, even brief periods of correlation, and may relay information on correlation state of the cortex.

The results of the 10 FS neuron network results were verified in a larger network of 125 FS neurons, corresponding to a cube of striatum with sides $0.5 \mathrm{~mm}$. To demonstrate correlation detection, only a group of 27 neurons $[3 \times 3 \times 3$ cube (Fig. $7 B$ ) $]$ were given correlated input during the $20 \mathrm{~ms}$ window; all other neurons continued to receive uncorrelated inputs. Similar to the results with the small network, a transient increase in synaptic input correlation produces a brief increase in firing for the gap junction coupled case (Fig. 7C). A cross-correlogram generated for various sized subnetworks within the larger 125 FS network shows that the synchronization is local. As pairs within an increasingly larger subset of the 125 FS network are included in the analysis the weak synchronization peak seen for directly connected neighbors decreases (Fig. 7D). This indicates that there is no global synchronization in the network. Another role proposed for gap junctions is to generate propagating waves of spikes in a network. Such wave activity is not seen in the FS network, because a spike 
A

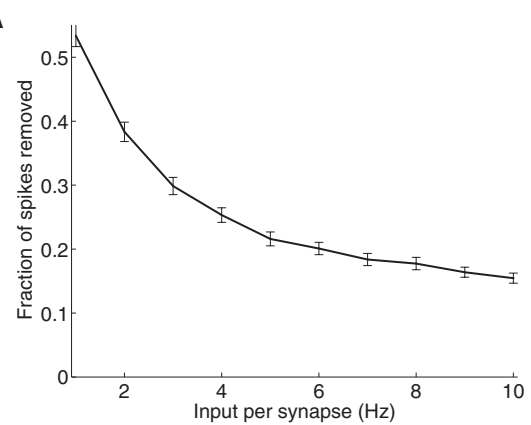

B

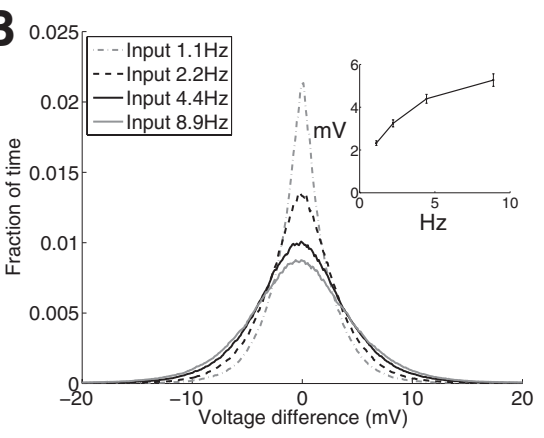

C

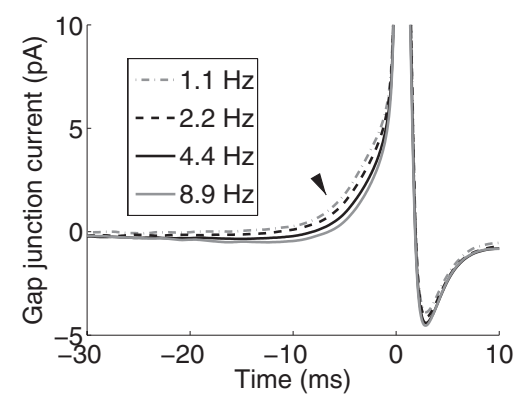

Figure 6. Spike frequency reduction decreases as synaptic activation increases in the network with gap junctions. $\boldsymbol{A}$, At successively higher synaptic activation frequency, a smaller fraction of the spikes is removed in the presence of gap junctions in the $10 \mathrm{FS}$ interneuron network. The fraction removed is the reduction in firing frequency in the network with gap junctions, divided by the firing frequency in the network devoid of gap junctions. $\boldsymbol{B}$, As the activation frequency per synapse increases (resulting in more spikes in the network), so does the average voltage difference across the gap junction (inset) and the distribution widens, giving rise to larger average currents across the gap junction. $C$, Despite the average increase in the current through the gap junction during the whole simulation, just before a spike the current through the gap junctions is slightly smaller for high input frequencies. This is because with increased synaptic activation frequency it is more likely that the electrically coupled neighbor is depolarized at the same time. This, together with the flattening shape of the $I-F$ curve (see Fig. $5 A$ ), explains the decreased reduction in spike frequencies for higher synaptic input frequencies. $\boldsymbol{D}$, Change in spike frequency caused by addition of gap junctions as a function of synaptic input activation frequency. Solid trace shows the shunted spikes (see also Fig. $4 A$ ), dashed line shows those appearing spontaneously (see also Fig. 4C), and dash-dotted line represents the spikes that are triggered by a neighboring neuron spike (see also Fig. 4B). The gray dotted trace shows that the net reduction in spikes levels off with higher input intensities. $\boldsymbol{E}$, Increased synaptic activation leads to higher relative spike synchronization. At the higher input, a spike in one neuron more easily triggers a synchronized spike in a neighboring neuron. Also the rate by which spikes are shunted away levels off. The synchrony in the network with gap junctions is higher than the shuffled control traces (where direct interactions mediated by gap junctions are removed). Spikes are considered synchronous if they occurred within $5 \mathrm{~ms}$ of each other. Note that even though the reference network devoid of gap junctions spikes more and thus by chance has higher occurrences of spike pairs, spike synchronization is even higher in the subpopulation of directly coupled neighbors despite a lower average firing frequency.

in a reference neuron is rarely followed by a spike in a gap junction coupled neighboring neuron. A spike in a striatal FS neuron is followed by $<0.2$ spikes within $10 \mathrm{~ms}$ in their gap junction connected neighbors for proximal gap junctions and 0.3 spikes within $10 \mathrm{~ms}$ for distal gap junctions (supplemental Fig. S1E, available at www.jneurosci.org as supplemental material). This lack of global synchronization or propagated activity is in accor- dance with the relatively sparse striatal gap junction connectivity (Galarreta and Hestrin, 2001b).

\section{Discussion}

A model network, corresponding to a striatal fast-spiking interneuron population projecting to one medium spiny neuron, was constructed to investigate the effect of gap junctions (electrical synapses) on the activity of the synaptically driven FS population. The model was based on a previously published FS interneuron model (Kotaleski et al., 2006).

The novel finding in this study is a reduction in firing frequency when synaptically driven FS interneurons in a network are coupled through gap junctions. Gap junctions do increase synchrony of FS interneuron firing, but this effect is modest compared with the spike frequency reduction when inputs to the network neurons are uncorrelated or even moderately correlated. This result leads to the prediction that simultaneously recorded FS interneurons do not spike synchronously. This prediction is supported by an in vivo study, showing little correlation between nearby FS neurons (Berke, 2008). The same group, using in vivo studies, further shows that the FS neurons are selective for direction and a large number of them are active only around the time of action selection (Gage et al., 2008). This result is consistent with the simulated increase in FS interneuron firing in response to transient input correlation from cortex. Such changes in cortical spike synchronization have been suggested to occur during motor planning (Riehle et al., 1997, 2000).

\section{Shunting reduces network activity}

Gap junctions reduce spiking activity in networks activated by synaptic input by shunting charge from more depolarized neurons to less depolarized neighbors. This is consistent with predictions in Amitai et al. (2002) as well as observations of increased cortical inhibitory currents in Cx36 knock-out mice (Cummings et al., 2008). The synaptic input together with activation of ionic conductances in the neurons drives the fluctuations in membrane potential and produce more action potentials than would constant current injection of the same mean. Due to these fluctuations, when one neuron is depolarized to spike threshold, the neighbor usually is not depolarized, leading to an increased outward current through the gap junctions from the more depolarized neuron (see also Fig. 5C). In essence, the effect of gap junctions is to shunt current to nonspiking connected neurons, lowering the firing frequency back to that observed with constant current injection of the same mean. 


\section{Spike synchronization is moderate} and local

Although the dominating effect of gap junctions is the removal of spikes, they can also add spikes to the electrically coupled neurons, leading to a moderate spike synchronization between the electrically coupled neurons. This is in accordance with other studies predicting spike synchronization (Traub et al., 2001; Amitai et al., 2002; Pfeuty et al., 2003; Gibson et al., 2005). Synchronization increases with gap junction conductance and with synaptic activation frequency, but remains modest with physiological values of coupling and synaptic inputs.

Synchronization appears to be a local effect mainly between directly coupled FS interneurons in the model; it is rarely seen for pairs of FS neurons which are indirectly coupled through their respective gap junctions to a shared neighbor. The lack of global synchrony, especially notable in the large network (supplemental Fig. S1D, available at www.jneurosci.org as supplemental material), is in contrast to the hippocampus where gap junctions above a certain threshold $(0.35 \mathrm{nS})$ in simulations promoted global synchronization in the gamma $(30-70 \mathrm{~Hz})$ range (Traub et al., 2001) when driven by current injections. Three factors may explain the difference between these two studies. One is the type of input: current injection versus synaptic stimulation. This study shows that synchrony is more prominent in response to current injection; thus perhaps global synchrony is observed more in response to current injection. The second factor is the size of the network: Schneidman et al. (2006) showed that in networks showing weak pairwise synchrony, strongly correlated states may emerge in large networks; however, no global synchrony emerged in the simulations with a larger striatal FS network of 125 neurons. A third factor is the presence of GABAergic synapses: Galarreta and Hestrin (2001a) found that gap junctions together with GABA synapses promoted increased synchronization in cortical FS interneuron networks. In contrast, FS interneurons in striatum rarely appear to be connected by GABAergic synapses (Koós and Tepper, 1999). Thus, any global synchronization in the striatum is likely a result of correlations in the input driving the network.

\section{Spatial correlation modulates effect of gap junctions}

The spike reduction due to gap junctions depends on spatial correlation of inputs to neighboring neurons. Correlations in the synaptic inputs between the FS interneurons reduce the shunting between electrically coupled neurons, and lead to increased output frequency only for the FS network with gap junctions. Spike frequency reduction due to shunting is not observed with constant current injections because this is an extreme form of spatially correlated input, where there are no temporal fluctuations either. Nonetheless, the effect of uncorrelated synaptic inputs can be imitated by providing unequal current injection to connected neurons (Fig. 5A). This unequal current injection creates voltage

\section{C}

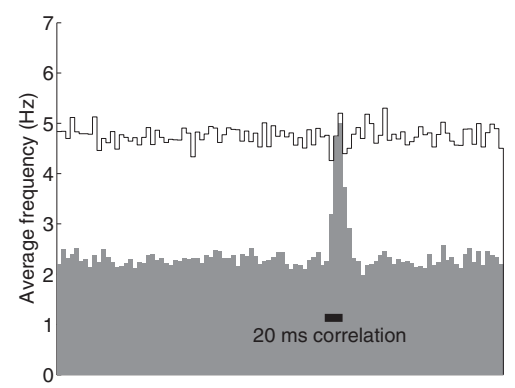

D

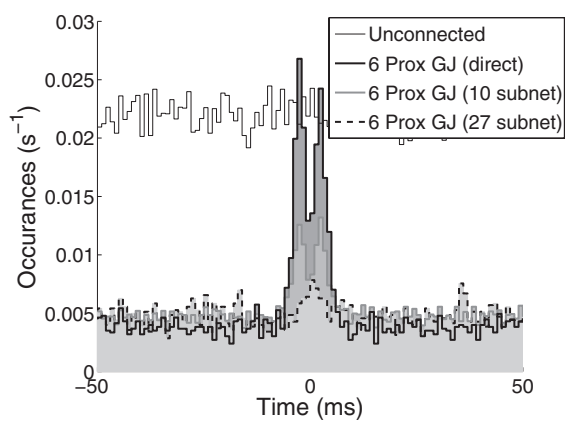


stead the somatic coupling coefficient is kept constant while moving the gap junctions more distally, by increasing the number of gap junctions (Hjorth et al., 2006), then the shunting instead increases and there is a larger spike reduction in the network. Small changes in the maximal conductance and activation and inactivation time constants of $\mathrm{Na}, \mathrm{K}_{\mathrm{A}}, \mathrm{Kv} 3.1 / 3.2$, and $\mathrm{Kv} 1.3$ channels alter the spike frequency without changing the general findings of moderate spike synchronization and significant shunting (see supplemental Fig. S5, available at www.jneurosci. org as supplemental material).

\section{Functional implications}

The present simulations predict that the activity of FS neurons in a connected network depends on the degree of synchronous activation of the synapses to the different network neurons. The shunting by gap junctions decreases the response to uncorrelated inputs, but shunting is minimal in response to correlated inputs. Thus, a simultaneous increase in the activity and correlation of cortical inputs will produce a significant increase in activity in an electrically connected FS network. In summary, striatal FS networks may act as population detectors of correlated input activity.

The importance of such gap junction effects may relate to FS interneuron modulation of MS neuron outputs. Experiments have shown that GABA input before an up state can increase, but in some cases slightly decrease, the time to first spike (Gustafson et al., 2006) in striatal MS neurons. Due to the depolarizing nature of GABA in MS neurons, GABA before a subthreshold current injection can increase the MS neuron spike probability (Bracci and Panzeri, 2006). Nonetheless, the more likely scenario is that firing of an FS interneuron delays firing in an MS neuron (Koós and Tepper, 1999), and multiple FS spikes are predicted to delay the MS neuron spike further. In action selection the FS neurons are activated earlier than nearby MS neurons that decrease their activity, consistent with the role of FS neurons suppressing MS neuron firing as well as unwanted behaviors (Gage et al., 2008). This control of the MS spike timing is important for synaptic plasticity (Kerr and Plenz, 2004; Fino et al., 2005; Pawlak and Kerr, 2008). The relative fraction of MS spikes removed due to activity in presynaptic FS neurons may be controlled not only by a change in synaptic drive but also by a transient increase in input correlation (see supplemental Fig. S6, available at www. jneurosci.org as supplemental material).

In addition to the control of striatal FS network activity resulting from a change in the intensity or synchronicity by which neighboring FS neurons are activated from the cortex, FS activity is likely also controlled by the GABAergic input from globus pallidus externa (GPe), which selectively targets striatal FS neurons (Bevan et al., 1998). The rich innervation from striatal MS neurons to GPe (Lévesque and Parent, 2005) makes the dynamics of this negative feedback loop ideal for future modeling studies. All these complementary mechanisms of controlling FS activity, and thus indirectly MS spiking, might be important during behavioral tasks (Berke, 2008; Shouno et al., 2009).

\section{References}

Aertsen AMHJ, Gerstein GL, Habib MK, Palm G (1989) Dynamics of neuronal firing correlation: modulation of "effective connectivity." J Neurophysiol 61:900-917.

Amitai Y, Gibson JR, Beierlein M, Patrick SL, Ho AM, Connors BW, Golomb D (2002) The spatial dimensions of electrically coupled networks of interneurons in the neocortex. J Neurosci 22:4142-4152.

Bar-Gad I, Morris G, Bergman H (2003) Information processing, dimen- sionality reduction and reinforcement learning in the basal ganglia. Prog Neurobiol 71:439-473.

Bennett BD, Bolam JP (1994) Synaptic input and output of parvalbuminimmunoreactive neurons in the neostriatum of the rat. Neuroscience 62:707-719.

Berke JD (2008) Uncoordinated firing rate changes of striatal fast-spiking interneurons during behavioral task performance. J Neurosci 28:10075-10080.

Bevan MD, Booth PAC, Eaton SA, Bolam JP (1998) Selective innervation of neostriatal interneurons by a subclass of neuron in the globus pallidus of the rat. J Neurosci 18:9438-9452.

Blackwell KT, Czubayko U, Plenz D (2003) Quantitative estimate of synaptic inputs to striatal neurons during up and down states in vitro. J Neurosci 23:9123-9132.

Bolam JP, Hanley JJ, Booth PAC, Bevan MD (2000) Synaptic organization of the basal ganglia. J Anat 196:527-542.

Bower JM and Beeman D (1998) The book of GENESIS: exploring realistic neural models with the GEneral NEural SImulation System. New York: Springer.

Bracci E, Panzeri S (2006) Excitatory GABAergic effects in striatal projection neurons. J Neurophysiol 95:1285-1290.

Chow CC, Kopell N (2000) Dynamics of spiking neurons with electrical coupling. Neural Comput 12:1643-1678.

Connors BW, Long MA (2004) Electrical synapses in the mammalian brain. Annu Rev Neurosci 27:393-418.

Cummings DM, Yamazaki I, Cepeda C, Paul DL, Levine MS (2008) Neuronal coupling via connexin36 contributes to spontaneous synaptic currents of striatal medium-sized spiny neurons. J Neurosci Res 86:2147-2158.

Ding J, Peterson JD, Surmeier DJ (2008) Corticostriatal and thalamostriatal synapses have distinctive properties. J Neurosci 28:6483-6492.

Fino E, Glowinski J, Venance L (2005) Bidirectional activity-dependent plasticity at corticostriatal synapses. J Neurosci 25:11279-11287.

Fino E, Deniau JM, Venance L (2008) Cell-specific spike-timing dependent plasticity on GABAergic and cholinergic interneurons in corticostriatal rat brain slices. J Physiol 586:265-282.

Fukuda T (2009) Network architecture of gap junction-coupled neuronal linkage in the striatum. J Neurosci 29:1235-1243.

Gage GJ, Churchill MJ, Berke JD (2008) Selective involvement of striatal fast spiking interneurons during the choice between two learned actions. Soc Neurosci Abstr 34:578.15.

Galarreta M, Hestrin S (1999) A network of fast-spiking cells in the neocortex connected by electrical synapses. Nature 402:72-75.

Galarreta M, Hestrin S (2001a) Spike transmission and synchrony detection in networks of GABAergic interneurons. Science 292:2295-2999.

Galarreta M, Hestrin S (2001b) Electrical synapses between GABA-releasing interneurons. Nat Rev Neurosci 2:425-433.

Galarreta M, Hestrin S (2002) Electrical and chemical synapses among parvalbumin fast-spiking GABAergic interneurons in adult mouse neocortex. Proc Natl Acad Sci U S A 99:12438-12443.

Gibson JR, Beierlein M, Connors BW (2005) Functional properties of electrical synapses between inhibitory interneurons of neocortical layer 4 . J Neurophysiol 93:467-480.

Graybiel AM (2005) The basal ganglia: learning new tricks and loving it. Curr Opin Neurobiol 15:638-644.

Gustafson N, Gireesh-Dharmaraj E, Czubayko U, Blackwell KT, Plenz D (2006) A comparative voltage and current-clamp analysis of feedback and feedforward synaptic transmission in the striatal microcircuit in vitro. J Neurophysiol 95:737-752.

Hjorth J, Elias AH, Kotaleski JH (2006) The significance of gap junction location in striatal fast spiking interneurons. Neurocomputing 70:1887-1891.

Kalanithi PSA, Zheng W, Kataoka Y, DiFiglia M, Grantz H, Saper CB, Schwartz ML, Leckman JF, Vaccarino FM (2005) Altered parvalbuminpositive neuron distribution in basal ganglia of individuals with Tourette syndrome. Proc Natl Acad Sci U S A 102:13307-13312.

Kasanetz F, Riquelme LA, Murer MG (2002) Disruption of the two-state membrane potential of striatal neurones during cortical desynchronisation in anaesthetised rats. J Physiol 543:577-589.

Kasanetz F, Riquelme LA, O’Donnell P, Murer MG (2006) Turning off cortical ensembles stops striatal up states and elicits phase perturbations in cortical and striatal slow oscillations in rat in vivo. J Physiol 577:97-113. 
Kawaguchi Y, Wilson CJ, Augood SJ, Emson PC (1995) Striatal interneurones: chemical, physiological and morphological characterization. Trends Neurosci 18:527-535.

Kerr JND, Plenz D (2004) Action potential timing determines dendritic calcium during striatal up-states. J Neurosci 24:877-885.

Kincaid AE, Zheng T, Wilson CJ (1998) Connectivity and convergence of single corticostriatal axons. J Neurosci 18:4722-4731.

Kitano K, Aoyagi T, Fukai T (2001) A possible functional organization of the corticostriatal input within the weakly-correlated striatal activity: a modeling study. Neurosci Res 40:87-96.

Koós T, Tepper JM (1999) Inhibitory control of neostriatal projection neurons by GABAergic interneurons. Nat Neurosci 2:467-472.

Koos T, Tepper JM, Wilson CJ (2004) Comparison of IPSCs evoked by spiny and fast-spiking neurons in the neostriatum. J Neurosci 24:7916-7922.

Kotaleski JH, Plenz D, Blackwell KT (2006) Using potassium currents to solve signal to noise problems in inhibitory feedforward networks of the striatum. J Neurophysiol 95:331-341.

Lévesque M, Parent A (2005) The striatofugal fiber system in primates: a reevaluation of its organization based on single-axon tracing studies. Proc Natl Acad Sci U S A 102:11888-11893.

Mahon S, Vautrelle N, Pezard L, Slaght SJ, Deniau JM, Chouvet G, Charpier S (2006) Distinct patterns of striatal medium spiny neuron activity during the natural sleep-wake cycle. J Neurosci 26:12587-12595.

Nomura M, Fukai T, Aoyagi T (2003) Synchrony of fast-spiking interneurons interconnected by GABAergic and electrical synapses. Neural Comput 15:2179-2198.

Palm G, Aertsen AMHJ, Gerstein GL (1988) On the significance of correlations among neuronal spike trains. Biol Cybern 59:1-11.

Pawlak V, Kerr JND (2008) Dopamine receptor activation is required for corticostriatal spike-timing-dependent plasticity. J Neurosci 28:2435-2446.

Pfeuty B, Mato G, Golomb D, Hansel D (2003) Electrical synapses and synchrony: the role of intrinsic currents. J Neurosci 23:6280-6294.

Plenz D, Kitai ST (1998) Up and down states in striatal medium spiny neurons simultaneously recorded with spontaneous activity in fast-spiking interneurons studied in cortex-striatum-substantia nigra organotypic cultures. J Neurosci 18:266-283.

Ramanathan S, Hanley JJ, Deniau JM, Bolam JP (2002) Synaptic convergence of motor and somatosensory cortical afferents onto GABAergic interneurons in the rat striatum. J Neurosci 22:8158-8169.
Reynolds JNJ, Wickens JR (2002) Dopamine-dependent plasticity of corticostriatal synapses. Neural Netw 15:507-521.

Riehle A, Grün S, Diesmann M, Aertsen A (1997) Spike synchronization and rate modulation differentially involved in motor cortical function. Science 278:1950-1953.

Riehle A, Grammont F, Diesmann M, Grün S (2000) Dynamical changes and temporal precision of synchronized spiking activity in monkey motor cortex during movement preparation. J Physiol Paris 94:569-582.

Rudolph M, Destexhe A (2001) Do neocortical pyramidal neurons display stochastic resonance? J Comput Neurosci 11:19-42.

Schneidman E, Berry MJ 2nd, Segev R, Bialek W (2006) Weak pairwise correlations imply strongly correlated network states in a neural population. Nature 440:1007-1012.

Schultz W (1998) Predictive reward signal of dopamine neurons. J Neurophysiol 80:1-27.

Shen W, Flajolet M, Greengard P, Surmeier DJ (2008) Dichotomous dopaminergic control of striatal synaptic plasticity. Science 321:848-851.

Shouno O, Takeuchi J, Tsujino H (2009) A spiking neuron model of the basal ganglia circuitry that can generate behavioral variability. In: The basal ganglia IX, Vol 58, Advances in behavioral biology. New York: Springer.

Stern EA, Jaeger D, Wilson CJ (1998) Membrane potential synchrony of simultaneously recorded striatal spiny neurons in vivo. Nature 394:475-478.

Tecuapetla F, Carrillo-Reid L, Bargas J, Galarraga E (2007) Dopaminergic modulation of short-term synaptic plasticity at striatal inhibitory synapses. Proc Natl Acad Sci U S A 104:10258-10263.

Tepper JM, Koós T, Wilson CJ (2004) GABAergic microcircuits in the neostriatum. Trends Neurosci 27:662-669.

Traub RD, Kopell N, Bibbig A, Buhl EH, LeBeau FEN, Whittington MA (2001) Gap junctions between interneuron dendrites can enhance synchrony of gamma oscillations in distributed networks. J Neurosci 21:9478-9486.

Wickens JR, Arbuthnott GW, Shindou T (2007) Simulation of GABA function in the basal ganglia: computational models of GABAergic mechanisms in basal ganglia function. Prog Brain Res 160:313-329.

Wilson CJ, Kawaguchi Y (1996) The origins of two-state spontaneous membrane potential fluctuations of neostriatal spiny neurons. J Neurosci 16: 2397-2410. 